\title{
Pandemic Food Delivery: Plummet or Prosper?
}

\section{Jared A. Coleman ${ }^{1} \&$ Natalie Carrillo ${ }^{2}$}

\author{
Herbert Wertheim College of Engineering ${ }^{1}$, Warrington College of Business ${ }^{2}$, University of Florida \\ Faculty Mentor: Anne Donnelly, Center for Undergraduate Research
}

\begin{abstract}
As one of the many unique markets where the opportunity arose to utilize the COVID-19 pandemic to stimulate monetary growth and increase popularity, third-party delivery services made an interesting case to evaluate financial performance pre- and mid-pandemic. The primary purpose of this research was to determine whether the businesses GrubHub, UberEats, and DoorDash were successful in facilitating growth with the seemingly advantageous circumstances provided by the national emergency. Financial performance was evaluated utilizing economic key performance indicators (KPIs) for revenue, liquidity, and profitability. All data sets were collected from eight quarterly income statements and balance sheets from 2019 and 2020 for each company. Averages of the KPIs were computed for cumulative quarters prior to and including March 2020, after the announcement of COVID-19 restrictions, and the quarters subsequent to that date range; the mean values were then compared across both time periods. Results suggest an overall positive trend for DoorDash for all metrics and negative trend in terms of liquidity for UberEats and GrubHub. Furthermore, revenues for all three platforms possessed an increased expansion rate during COVID-19 within a range of 9.07-12.60\%. Implications of this analysis include possible research of non-food delivery platforms that operate based on the sale of virtual products during the pandemic or further exploration of such companies as COVID-19 begins to subside.
\end{abstract}

Keywords: COVID-19, food delivery, GrubHub, UberEats, DoorDash, pandemic

\section{Introduction and Review of Literature}

The COVID-19 pandemic has gravely impacted large and small businesses alike across the world and, more specifically, in the United States. Smartphone technologies and social media were essential in helping Americans maintain connectivity in times with strict social distancing measures and of an abundance of fear of social and physical in-person contact. One domain of business which was deeply impacted by the presence of this pandemic was and remains to be restaurants, which were closed to in-person seating and some even closed to any service provision, during nationwide lockdowns. For the restaurants which remained open, the only two options for connecting paying customers with their goods were to allow for either takeout or 
delivery. Third-party delivery services are a special case to evaluate as they possessed a path which could use the pandemic as a stimulant for company popularity and growth.

Literature pertaining to this topic reveals a lack of analysis across these leading delivery services regarding the comparison of pre-COVID-19 marketing successes against mid-COVID19 marketing successes. Trends were found in marketing which pertained to a need for vendors as well as delivery services to have and maintain social media platforms in a technologically advanced society (Saravanakumar \& SuganthaLakshmi, 2012). The way in which marketing can reap success/lead to purchases via use of these platforms revolves around understanding consumer demand and presentation of the "WOW! factor", the signature aspect of the platform that engages the customer, coupled with simplicity in design of advertisements (Kotler et al., 2017). A visually aesthetic, quick advertisement with attention grabbing qualities tends to resonate with individuals, especially during a time of collective stress. Moreover, hedonic motivation plays a major role for food delivery services. Platforms that offer fun and pleasure are more likely to yield positive attitudes and positive customer attribution (Yeo et al., 2017).

In addition, the consumer demand for different domains of food has altered. In recent years, the attraction of healthy food options has become much more prevalent, with changes attributed primarily to marketing and menu content (Leone et al., 2020). Nonetheless, the unorthodox events surrounding the proliferating illness have recently flipped this trend. It was found that during the 13th and 14th weekends studied in the pandemic, unhealthy food prevailed over healthy food in terms of volume of deliveries in response to $95 \%$ of food delivery service advertisements portraying "sandwiches, ultra-processed beverages, traditional meals or pasta and pizzas" (Horta et al., 2020). The variety of different food types is still sought after, yet there is an imbalance in which type of food holds a heavier interest. To maintain a broader outreach, delivery services offering a larger variety of food vendors, healthy and unhealthy alike, now retain a greater degree of interest among possible consumers.

In the delivery sect, demand and resulting financial success varies among different types of households. A study on India's urban population (a population of interest for the topic because of the popularity of delivery food service in these cities) determined that single person households require certain standards in delivery and vendor services to be met in order to pique interest and initiate purchase (Thamaraiselvan et al., 2019). Evaluation of the relationship between food delivery services and single person households versus family households 
highlighted the necessity of cheaper alternative accommodations through delivery apps for a single person household (Cho et al., 2019). Therefore, financial well-being on both ends of the service spectrum must revolve around making prices affordable and offering a wide variety of options that is overwhelmingly apparent to potential consumers.

It is also worth noting the change in consumer behavior as news of COVID-19 rapidly spread. During the first week of COVID-19, there was an increase over $75 \%$ in the number of Google searches for "food shortage" as people panicked about being able to get food from grocery stores (Schmidt et al., 2020). Interest in food delivery services peaked as people found comfort in cooked food being delivered rather than worrying about going to a grocery store with potentially empty shelves. However, a new layer of trust in these delivery and vendor services had to be established before they were able to flourish successfully amidst the pandemic (Hobbs, 2020). Consumer interest was piqued through the utilization of COVID specific guidelines and their marketing to assuage consumer trust in product and handling. While this strategy was considered imperative for platform success, a study conducted on Chinese consumers named satisfaction to still be of the highest importance of participation in ordering food delivery, maintaining that quality and variety in food services both remain to be held of utmost interest to consumers, even during the pandemic (Cho et al., 2019). The basis of this investigation rests on the recent explosion of online-to-offline business models in China, a phenomenon that has been well underway in the U.S. As such, similar results in the U.S. are expected. Alongside satisfaction, task-technology fit and confirmation are among the top three determiners for intended reuse of food delivery applications during COVID-19. If consumers perceive that the apps fulfill their daily necessities in a technologically simple manner and that their current experience confirms their expectations, their utilization of the companies surges (Zhao \& Bacao, 2020).

Although previous literature recognizes the way in which delivery services use technology to make their platforms relevant and the useful nature which these delivery services played in providing people meals during the pandemic, their focus is how delivery services impacted the pandemic rather than how the pandemic impacted delivery services. The purpose of this research is to evaluate whether the circumstances resulting from COVID-19 were able to improve the popularity and stimulate growth for three third-party delivery services: GrubHub, UberEats, and DoorDash. It is hypothesized that the three companies would have witnessed an 
expansion in sustained financial growth in the quarters which the pandemic coincided as compared to those previous.

\section{Design and Methods}

The financial health of the top three U.S. online food delivery shareholders GrubHub, UberEats, and DoorDash was measured using information provided by quarterly financial statements publicly available on company pages as required by the U.S. Securities and Exchange Commission. In particular, both the income statements and balance sheets of the businesses displayed considerable economic metrics, including revenues, expenses, assets, liabilities, cash and cash equivalents, accounts receivables, and net income. KPIs were utilized to supply a more detailed analysis of the companies beyond simple surface tracking of the accounting values discovered from the documents.

For profitability, two metrics-- gross profit margin (GPM) and net profit margin (NPM)-were calculated in order to determine the percentage of revenue remaining after the cost of goods sold were accounted for as well as the percentage of the revenue and other forms of income left subsequent to subtracting all other business expenses; the former margin is especially effective in exhibiting the profit generated by an item line, product, or certain service while the latter margin sufficiently explains the data in a more general sense, factoring in operating expenses, taxes, and interest accumulated by the company. In terms of liquidity, it is financially favorable if an entity can convert an asset into cash without drastically altering its market price. To capture this aspect, current and quick ratios were employed; the current ratio, comprised of the proportion of each

firm's current assets to current liabilities, indicates whether or not a firm is able to repay short term obligations within its accounting cycle; the quick ratio, on the other hand, measures a similar metric but only encompasses highly liquid assets such as cash and receivables. Working capital for each platform was additionally computed to indicate the operating liquidity it has on a day-to-day basis, rather than just quarterly ones that the previous ratios emphasize. Revenues were also included to demonstrate returns before manipulation of raw figures.

The performance of each of the food delivery applications was evaluated individually as a function of time in relation to the announcement of widespread closures as a result of the COVID-19 pandemic. Data was collected for each of the four three-month accounting quarters concluding on March 31, June 30, September 30, and December 31 for the years 2019 and 2020, 
in order to formulate an appreciable trend. In regard to the pandemic, nationwide business shutdowns began taking effect approximately two weeks into the month of March 2020. Accordingly, any values prior to the conclusion of the first quarter of 2020 were considered in this analysis to be pre-pandemic while the remaining three quarters were termed mid-pandemic. Considering that the outbreak is currently still occurring, the term mid-pandemic encompasses all dates since April 1, 2020 up to the last available financial quarter concluding on December 31, 2020. Pre-pandemic, therefore, includes any dates from January 1, 2019 to March 31, 2020. Yearly financial sources were excluded from this study as they did not provide a complete delineation of the effects yielded by the virus; only the final year analyzed would share potentially differing information as a single data point. For each firm, the KPIs were averaged based on their position on the pre-pandemic/mid-pandemic timeline; the difference between the two periods were taken and utilized to indicate a net positive or net negative change. All values were calculated to two significant digits after the whole number amount to preserve consistency.

As a subset of the larger conglomerate Uber Technologies, UberEats shares certain amounts with its parent company based on rides and freight orders (commercial shipments by Uber Technologies on behalf of other companies). This would usually pose a potential obstacle in placing the subset in the same industry as GrubHub and DoorDash. Nonetheless, since the research question seeks to evaluate the financial health of the three companies in relation to the disease outbreak, a comparative analysis was not undertaken. This prevents placing the firms in competition with one another and allows us to measure directions independent of such limiting factors.

\section{Results}

After exporting all data for the financial performance of the three companies and applying the appropriate KPI formulas, the following results were ascertained. Figure 1 exhibits the quarterly revenues for the platforms and Figures 2 and 3 showcase the quarterly GPMs and NPMs respectively. Figures 4-6 include the liquidity KPIs for each quarter; Figure 4 shows the current ratios, Figure 5 the quick ratios, and Figure 6 the working capital amounts for the businesses. The dashed line in all six figures indicates the dividing line between pre- and midpandemic. Table 1 shows all the averages discussed below. 
GrubHub's reported earnings pre-pandemic possessed an average value of \$335 million and a mid-pandemic value of $\$ 486$ million; the revenue growth rate for the two time periods was approximately $2.95 \%$ and $12.02 \%$ respectively, yielding a change of $+9.07 \%$. In terms of profitability, average GPM shrunk from $6.03 \%$ to $0.28 \%$ between the two time frames and average NPM shrunk from $-2.90 \%$ to $-25.21 \%$. Similarly, the mean current ratio for the company fell from 2.21 to 1.64 and mean quick ratio fell from 1.98 to 1.37. Average working capital for GrubHub pre-pandemic was about \$310 million and \$234 million mid-pandemic; the average change in working capital was $48.93 \%$ and $-19.47 \%$ respectively, dropping by $-68.40 \%$ across the periods.

Since UberEats is a subset of its larger parent company Uber Technologies, financial amounts for profitability and liquidity KPIs included that of the parent company. All aspects of the parent company uniformly responded to COVID-19 restrictions, thus influence from the other subsets on reported figures is likely minimal. Although Uber Technologies eventually transitioned to sharing specific income statements and balance sheet items for UberEats in the later quarters of 2020, those from Uber Technologies were employed throughout to avoid inconsistency. Revenues for UberEats as a sole entity were also included on the accounting records and, as a result, were examined only when comparing quarterly revenues and not used for KPIs. Earnings pre-pandemic averaged about \$666 million and about \$1339 million midpandemic; revenue growth rate expanded from $11.20 \%$ to $20.38 \%$, yielding a change of $+9.18 \%$. Average GPM rose from $-57.64 \%$ to $-39.95 \%$ and NPM from $-68.13 \%$ to $-48.28 \%$. Mean current ratio and mean quick ratio both decreased from pre-pandemic to mid-pandemic; mean current ratio from 2.24 to 1.48 and mean quick ratio from 1.97 to 1.08 . Likewise, average working capital experienced a drop for Uber Technologies, from \$6899 million to \$3093 million. The percentages in this change were approximately $30.58 \%$ and $-9.57 \%$, resulting in a net change of $-40.15 \%$.

Of the three third-party platforms, DoorDash possessed the largest revenue change from quarters prior to March 2020 to those subsequent. Average revenue pre-pandemic amounted to $\$ 249$ million and $\$ 841$ million mid-pandemic; revenue growth rate increased by $+12.60 \%$, from $29.74 \%$ to $42.35 \%$. Profitability KPIs had a similar outcome. Average GPM expanded from $40.66 \%$ to $53.37 \%$ and average NPM grew from $-75.24 \%$ to $-5.10 \%$. Liquidity for DoorDash only included mean current and quick ratios, where mean current ratio ballooned from 2.61 to 
3.10 and mean quick ratio from 0.82 to 2.28 . DoorDash's recent filing to become a public company in the last year has allowed most financial data to be readily available. However, certain income statement and balance sheet amounts from quarters of 2019 and the first quarter of 2020 are still unavailable as a result of DoorDash's previous private status. Thus, the necessary amounts for working capital could not be acquired for certain quarters and averaged to yield a percentage change for the pre-pandemic time frame. The overall trend for working capital is nonetheless visible in Figure 6.

\section{Figure 1}

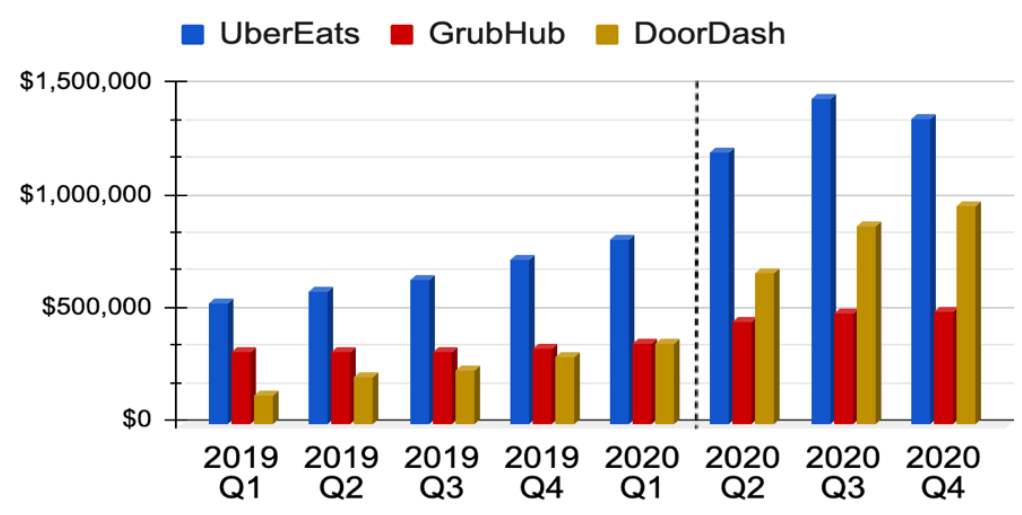

Revenues for each quarter (in thousands)

Figure 2

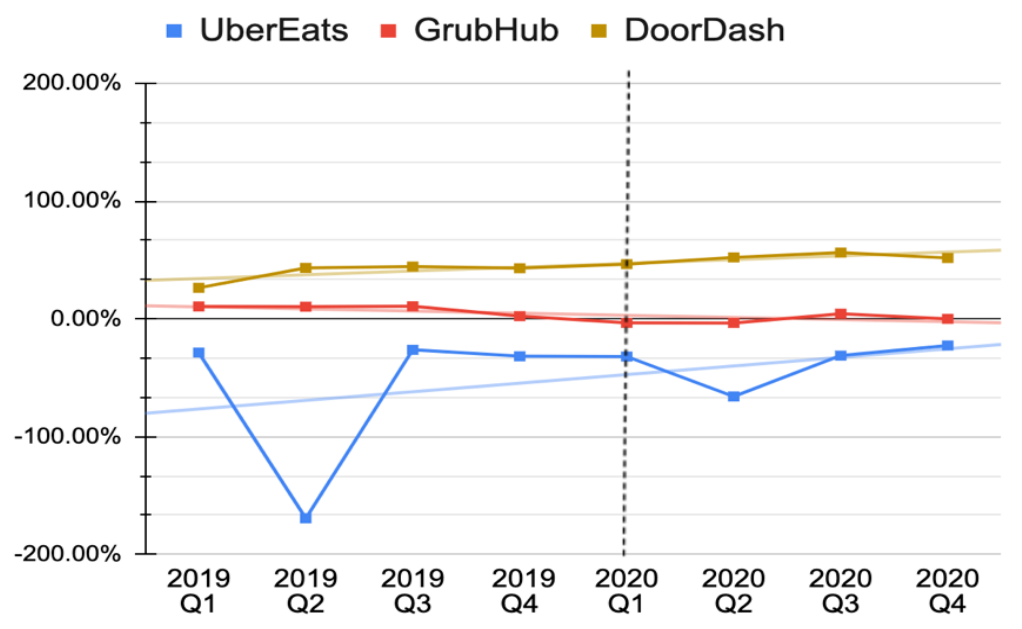

Gross profit margins (GPMs) for each quarter 
Figure 3

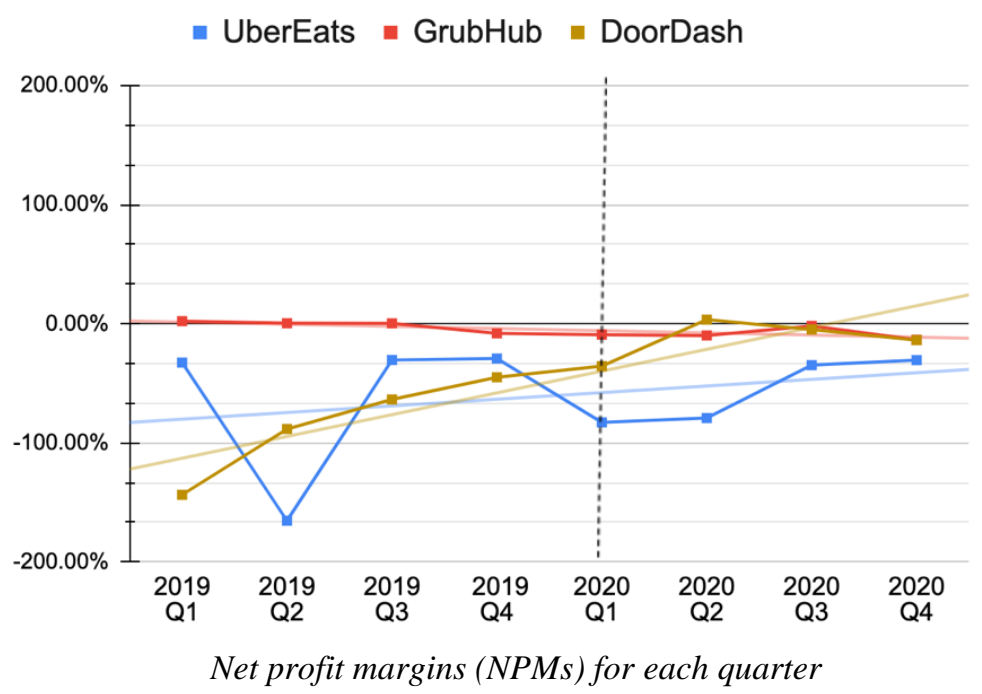

Figure 4

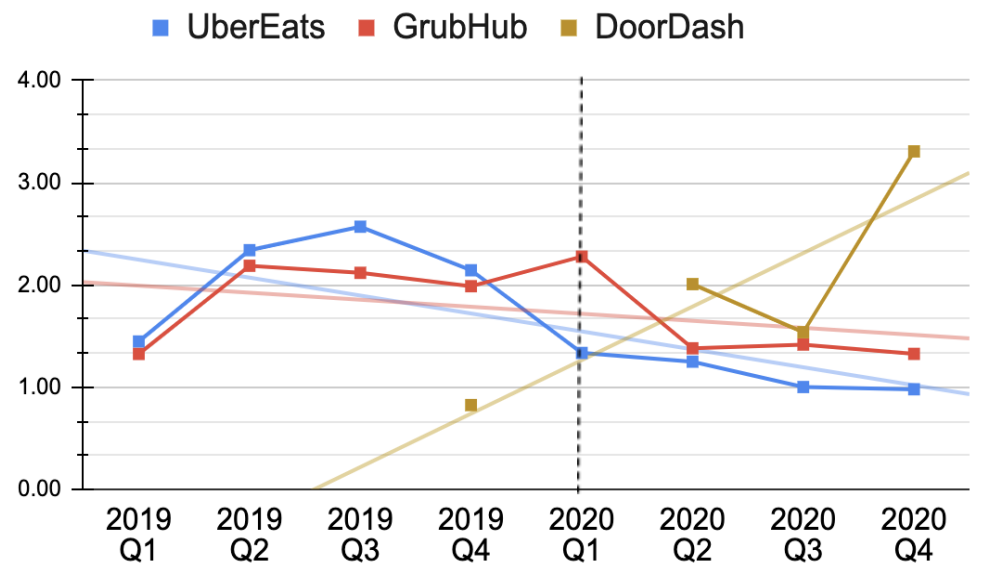

Current ratios for each quarter

Figure 5

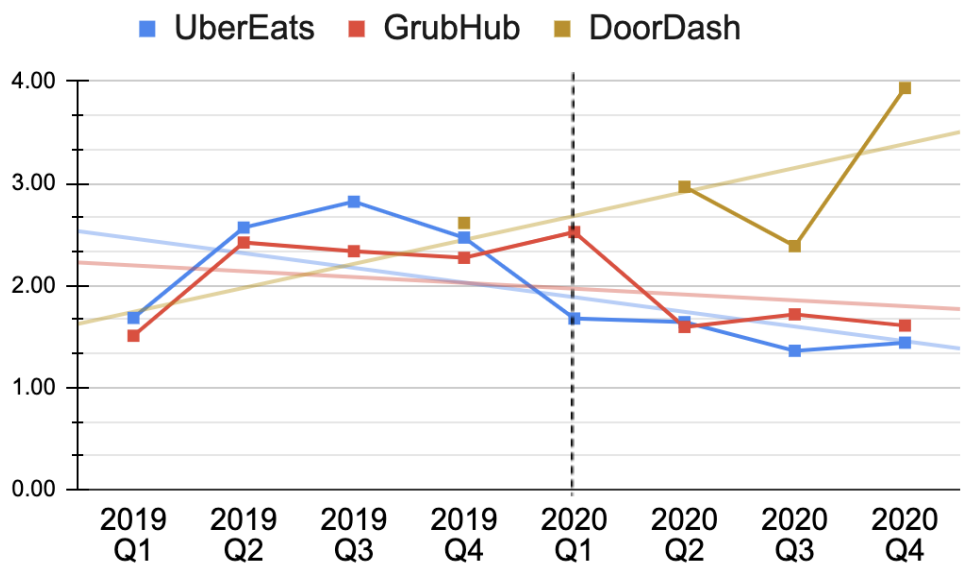

Quick ratios for each quarter 


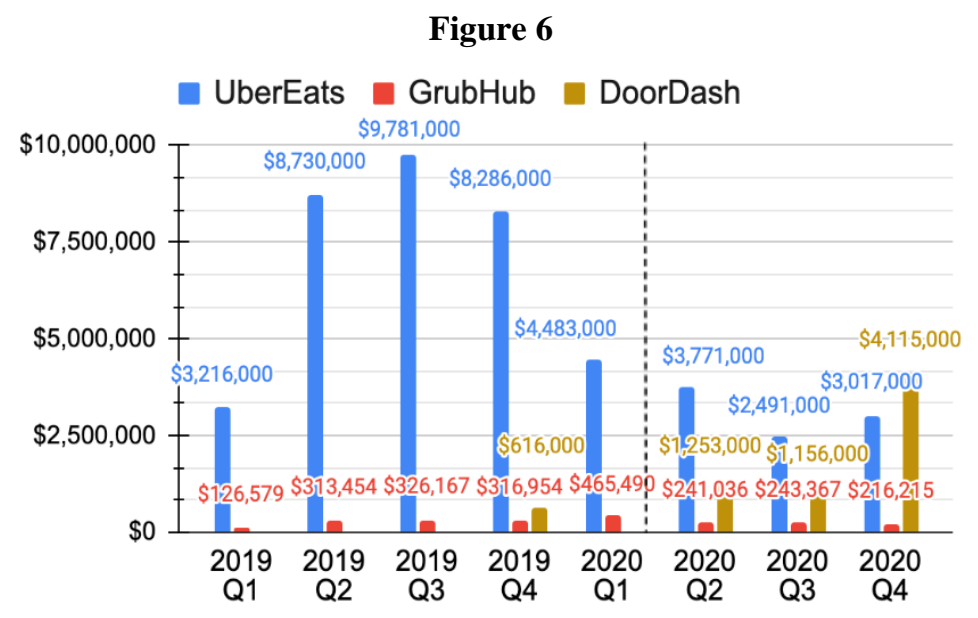

Working capital for each quarter (in thousands)

Table 1. Average Performance of Third-Party Food Delivery Platforms

\begin{tabular}{lcccccc}
\hline Financial Metrics & \multicolumn{3}{c}{ Pre-pandemic KPIs } & \multicolumn{3}{c}{ Mid-pandemic KPIs } \\
\hline & GrubHub & UberEats & DoorDash & GrubHub & UberEats & DoorDash \\
Avg. Revenue & $2.95 \%$ & $11.20 \%$ & $29.74 \%$ & $12.02 \%$ & $20.38 \%$ & $42.35 \%$ \\
Growth Rate & $6.03 \%$ & $-57.64 \%$ & $40.66 \%$ & $0.28 \%$ & $-39.95 \%$ & $53.37 \%$ \\
Avg. GPM & $-2.90 \%$ & $-68.13 \%$ & $-75.24 \%$ & $-25.21 \%$ & $-48.20 \%$ & $-5.10 \%$ \\
Avg. NPM & 2.21 & 2.24 & 2.61 & 1.64 & 1.48 & 3.10 \\
Mean Current Ratio & 1.98 & 1.97 & 0.82 & 1.37 & 1.08 & 2.28 \\
Mean Quick Ratio & $30.58 \%$ & $48.93 \%$ & -- & $-9.57 \%$ & $-19.47 \%$ & $124.11 \%$ \\
Avg. Change in & & & & & & \\
Working Capital & & & & & &
\end{tabular}

Table 2. Differences in Average Performance

\begin{tabular}{lccc}
\hline Financial Metrics & \multicolumn{3}{c}{ Numerical Difference } \\
\hline & GrubHub & UberEats & DoorDash \\
Avg. Revenue Growth Rate & $+9.07 \%$ & $+9.18 \%$ & $+12.60 \%$ \\
Avg. GPM & $-5.75 \%$ & $+17.68 \%$ & $+12.71 \%$ \\
Avg. NPM & $-22.31 \%$ & $+19.93 \%$ & $+70.14 \%$ \\
Mean Current Ratio & -0.57 & -0.76 & +0.48 \\
Mean Quick Ratio & -0.61 & -0.89 & +1.46 \\
Avg. Change in Working Capital & $-68.40 \%$ & $-40.15 \%$ & -- \\
\hline
\end{tabular}




\section{Discussion and Conclusions}

Although not all segments of the firms' economic success could be evaluated and examined as a result of time constraints, the computed KPIs supply an elementary indication. Reviewing the revenues of the platforms, all three experienced a net positive change in the growth rate of its earnings. DoorDash possessed the largest change, approximately $12.60 \%$, followed by UberEats at $9.18 \%$ and GrubHub at 9.07\%, as shown in Table 2. In terms of profitability, GrubHub saw a negative shift in both GPM and NPM, while both UberEats and DoorDash had higher GPMs and NPMs after the institution of COVID-19 restrictions (Table 2). The liquidity for GrubHub and UberEats dropped an appreciable amount as evidenced by the decrease in both the current ratio and quick ratio from pre- to mid-pandemic quarters (Table 2). Average change in working capital was also lower in the mid-pandemic time period for the two companies than prior (Table 2). DoorDash, on the other hand, became more liquid over time as shown by the increase in both its current and quick ratios, also visible in Table 2. The change for DoorDash in working capital was excluded due to absence of aforementioned data.

It is likely that the three businesses enjoyed a boost in revenues between the prepandemic and mid-pandemic time periods due to the simultaneous overlap of closure of inperson seating for many restaurants and shelter-in-place orders; evidently, this likely caused many businesses to heavily rely on the delivery services as a means of transport and many customers to prefer food drop-off to food pick-up. This trend partially agrees with the hypothesis that the firms would become more profitable over time as a result of COVID-19; the firms acquired more money than they usually do under normal circumstances. However, the sustainability of this growth that was hypothesized does not seem to be fully corroborated. For example, GrubHub and UberEats both became less liquid over time. This suggests that current liabilities grew at a faster rate than assets such as cash and receivables, acknowledging the companies acquired more debt while earning more. Evidently, such a phenomenon appears to be confirmed when examining the companies' financial statements. GrubHub additionally saw a drop in profitability. Both of these phenomena are likely due to increased costs to operate that accompanied increased earnings. Nonetheless, DoorDash improved in all areas measured. This could be due to a number of variables including those mentioned above, anticipation in expansion by investors after its announcement of transition to becoming public, or other actions such as its decision to cut restaurant commissions and acquisition of smaller businesses such as 
Caviar. Moreover, it is more than possible that the occupation of all three platforms in the same industry naturally induced competition that meant the success of one company at the expense of another. A limitation to this argument includes the proposition that the three companies are not true competitors as they do not operate in the same geographical locations; DoorDash is available only in the U.S., Canada, and Australia, while UberEats and GrubHub additionally offer services in South America, Western Europe, and Eastern Asia. Nonetheless, the three businesses are primarily governed in the U.S. and share similar regulations, preventing any from truly functioning outside the domain of the others.

The long-term implications on such an analysis encompass wide-ranging topics from consumer behavior to retailer strategies. Evidently, the COVID-19 pandemic acts as a scenario in which large scale traditional economic operations are either partially or entirely disrupted, creating a challenge for businesses to discover novel methods to continue providing services to paying customers. Third-party food delivery platforms, which are still in relative infancy, provide a perfect subject of study as their dependence on mobile applications and online systems as well as their vulnerability to the financial instability of vendor and consumer relations which they mediate create an interesting situation; increased presence on applications and decreased purchasing volume due to physical restrictions have opposite effects that demonstrate how such an economic sector will react in similar interruption events. This information is indispensable as it allows for financial planning for platforms whose main source of revenue comes from providing a service that many companies can eventually assume on their own.

Moreover, this research paves the way for observation of other businesses that market products that do not necessarily require physical contact to purchase. For example, the video game industry and media-streaming services possess the ability to continue generating revenue despite COVID-19 restrictions; further study of these companies can demonstrate appreciable consumer trends and interests as they develop throughout this pandemic.

\section{Acknowledgements}

Many thanks to Dr. Anne Donnelly for providing guidance and feedback for various steps of the research process. Also, a special recognition to Colby Tomasello for advice on data analysis and organization of the scheduling timeline. 


\section{References}

Cho, M., Bonn, M. A., \& Li, J. J. (2019). Differences in perceptions about food delivery apps between single-person and multi-person households. International Journal of Hospitality Management, 77, 108-116. https://doi.org/10.1016/j.ijhm.2018.06.019

Hobbs, J. E. (2020). Food supply chains during the COVID-19 pandemic. Canadian Journal of Agricultural Economics/Revue Canadienne d'agroeconomie, 68(2), 171-

176. https://doi.org/10.1111/cjag.12237

Horta, P. M., de Paula Matos, J., \& Mendes, L. L. (2020). Digital food environment during the coronavirus disease 2019 (COVID-19) pandemic in Brazil: an analysis of food advertising in an online food delivery platform. British Journal of Nutrition, 1-6.

https://doi.org/10.1017/S0007114520004560

Kotler, P., Kartajaya, H., \& Setiawan, I. (2017). Marketing 4.0: Moving from traditional to digital. Hoboken, NJ: Wiley.

Leone, L. A., Fleischhacker, S., Anderson-Steeves, B., Harper, K., Winkler, M., Racine, E., ... \& Gittelsohn, J. (2020). Healthy food retail during the COVID-19 pandemic: Challenges and future directions. International Journal of Environmental Research and Public Health, 17(20). https://doi.org/10.3390/ijerph17207397

Saravanakumar, M., \& SuganthaLakshmi, T. (2012). Social media marketing. Life Science Journal, 9(4), 4444-4451. http://www.lifesciencesite.com/lsj/life0904/670_13061life0904_4444_4451

Schmidt, C., Goetz, S., Rocker, S., \& Tian, Z. (2020). Google searches reveal changing consumer food sourcing in the COVID-19 pandemic. Journal of Agriculture, Food Systems, and Community Development, 9(3), 1-8. https://doi.org/10.5304/jafscd.2020.093.032 
Thamaraiselvan, N., Jayadevan, G. R., \& Chandrasekar, K. S. (2019). Digital food delivery apps revolutionizing food products marketing in India. International Journal of Recent Technology and Engineering, 8, 662-665. https://doi.org/10.35940/ijrte.B1126.0782S619

Yeo, V. C. S., Goh, S. K., \& Rezaei, S. (2017). Consumer experiences, attitude and behavioral intention toward online food delivery (OFD) services. Journal of Retailing and Consumer Services, 35, 150-162. https://doi.org/10.1016/j.jretconser.2016.12.013

Zhao, Y., \& Bacao, F. (2020). What factors determining customer continuingly using food delivery apps during 2019 novel coronavirus pandemic period? International Journal of Hospitality Management, 91, 102683. https://doi.org/10.1016/j.ijhm.2020.102683 